\title{
DIMENSIÓN INTERCULTURAL EN LA ENSEÑANZA DE LAS LENGUAS Y FORMACIÓN DEL PROFESORADO
}

\author{
María Silvina Paricio \\ Instituto de Enseñanza Secundaria Someso, La Coruña, España
}

\section{INTRODUCCIÓN}

La enseñanza y el aprendizaje de las lenguas extranjeras han de ponerse siempre en relación con el contexto social, político, económico y cultural en el que tienen lugar. Éste influye de manera determinante en el enfoque a adoptar, los métodos a seguir, etc. No cabe duda de que el nuevo escenario de la Unión Europea, e incluso el más amplio de la globalización de los intercambios económicos a nivel mundial, están teniendo repercusiones en el modo de abordar la enseñanza de las lenguas, orientando esta última hacia la dimensión intercultural. Ello implica el planteamiento de nuevos desafíos para el profesorado del área, al que se atribuyen nuevos roles y nuevas tareas a los que tendrá que hacer frente en su quehacer cotidiano.

Expondremos primeramente a grandes rasgos cuáles son las directrices europeas en materia de enseñanza de lenguas para centrarnos a continuación en los cambios derivados del manifiesto deseo de potenciar la dimensión intercultural. Nos detendremos así en definir los pilares básicos en los que se asienta el enfoque intercultural aplicado a la enseñanza de las lenguas, explicando cuáles son los componentes de la dimensión cultural y en qué consisten las nociones de competencia comunicativa intercultural y de conciencia cultural crítica, dos conceptos clave en esta nueva orientación. Dedicaremos asimismo un apartado a exponer cuál debe ser el papel del profesorado en el desarrollo de la dimensión intercultural, concluyendo con un conjunto de pautas encaminadas a orientar su formación inicial y continua en esa dirección.

El enfoque intercultural se ve apoyado por los más recientes desarrollos en el campo de la didáctica de las lenguas, que han abierto nuevas vías al apuntar hacia una enseñanza más integrada de lengua y cultura (Buttjes y Byram, 1991; Byram, 1997; Byram y Risager, 1999; Byram y Fleming, 2001; Kramsch, 1993; Zarate, 1986). Estos desarrollos empiezan a dejar su huella en las directrices establecidas por las Administraciones Educativas en los currículos de lenguas extranjeras de los estados miembros de la Unión. Un estudio sobre la enseñanza de las lenguas en entornos escolares en Europa, llevado a cabo por Eurydice (2001), pone de manifiesto que los programas oficiales de la mayor parte de los países europeos, además de optar masivamente por el enfoque comunicativo, incluyen el componente cultural como una dimensión importante. Según se recoge en los mencionados documentos, a través del aprendizaje de lenguas, el alumnado debe ampliar su conocimiento y profundizar en la comprensión de las poblaciones que las hablan, de sus costumbres y modos de vida. Estos objetivos formulados en términos de apertura al otro se acompañan de la invitación al ejercicio de una reflexión personal sobre la propia cultura. Son varios también los programas que consideran el conocimiento de una o varias lenguas extranjeras como un factor de enriquecimiento personal y profesional. 
La publicación, en el 2001, del Consejo de Europa del Marco de referencia europeo para el aprendizaje, la enseñanza, y la evaluación de las lenguas (Instituto Cervantes, 2002) ${ }^{1}$ ha supuesto un importante avance de cara a la planificación de la enseñanza de las lenguas en Europa. El documento, que concede un papel importante a la dimensión intercultural, refleja el deseo de esta entidad de adaptar la enseñanza de idiomas a las necesidades del mundo contemporáneo y de desarrollar consensos sobre los objetivos y principios por los que ha de guiarse dicha enseñanza en el futuro. Concebido como una especie de guía, su finalidad es proporcionar un conjunto de orientaciones en cuanto a objetivos, metodología, procedimientos de evaluación, etc., de utilidad para administraciones educativas, autores de libros de texto, formadores de formadores y profesorado en general.

\section{LA NORMATIVA COMUNITARIA EN MATERIA DE POLIITICA LINGÜÍSTICA}

Dentro de la política educativa europea, el aprendizaje de lenguas ocupa ya desde hace años un lugar importante. Tanto el Consejo de Europa como la Unión Europea asumen en sus textos oficiales una postura de defensa abierta del plurilingüismo y colaboran activamente en la adopción de medidas concretas en ese terreno. Ambas entidades consideran la diversidad lingüística y cultural de Europa como un rico patrimonio que es necesario preservar. Además, el conocimiento de lenguas se presenta como un instrumento que favorece el mejor entendimiento entre los pueblos y la comprensión mutua, al tiempo que posibilita el desarrollo de actitudes de tolerancia y respeto hacia otras culturas, contribuyendo así a la creación de un sentimiento de ciudadanía europea. Junto con este discurso de corte humanista, el objetivo último que justifica el fomento del aprendizaje de lenguas -tanto en el entorno escolar como fuera de él- se encuentra en realidad en la necesidad de preparar a la ciudadanía europea para una creciente movilidad transnacional, consecuencia de la libre circulación de personas. El examen de los textos comunitarios permite seguir la pista a ese discurso que vincula el conocimiento de idiomas con la educación intercultural.

En el caso de la Unión Europea, la mención a la necesidad de promover el conocimiento de las lenguas comunitarias en los estados miembros figura ya en el artículo 126 del Tratado de Maastricht (actual 149 de la versión consolidada del Tratado de la Unión, Comisión Europea, 1999), que contempla como objetivo "desarrollar la dimensión europea de la enseñanza, especialmente a través del aprendizaje y de la difusión de las lenguas de los Estados miembros". Este mismo objetivo reaparece en el Libro verde sobre la dimensión europea de la educación (Comisión Europea, 1993). Remontándonos unos treinta años atrás, cabe reseñar la Resolución del Consejo y de los Ministros de Educación correspondiente al Consejo celebrado el 9 de febrero de 1976 (DO № C O38, 19-2-1976), que constituye prácticamente el primer texto oficial donde se contempla un programa de acción comunitario específico para el campo de la educación, incluyéndose un cierto número de objetivos relacionados con la enseñanza de lenguas en el sistema educativo y fuera de él. Se habla ya aquí -entre otras cuestionesde la conveniencia de promover intercambios de profesorado y alumnado, así como estancias en el extranjero -germen de los actuales programas Sócrates y Leonardo- y se apela a la necesidad de examinar a nivel comunitario las investigaciones en materia de metodología de la enseñanza de idiomas, especialmente los trabajos del Consejo de Europa.

\footnotetext{
${ }^{1}$ El documento original se publicó en el año 2001 en inglés y francés.
} 
El Libro blanco sobre la educación y la formación. Enseñar y aprender. Hacia la sociedad del conocimiento (Comisión Europea, 1995) incluye como uno de los objetivos educativos para construir la sociedad del conocimiento el "dominar tres lenguas comunitarias", objetivo quizá optimista en exceso. El documento alude a la firme voluntad de superar el carácter elitista que poseía antes el aprendizaje de idiomas, reservado generalmente a las clases sociales más favorecidas. Se plantea así la necesidad de posibilitar que todos los ciudadanos y ciudadanas europeos (no sólo los que están en edad escolar), con independencia de su nivel de formación o del itinerario educativo que escojan, adquieran y mantengan la capacidad de comunicarse al menos en dos lenguas extranjeras, además de la propia. La formulación de este objetivo tendría su origen en la Resolución del Consejo de Ministros de Educación, celebrado el 31 de marzo de 1995, sobre la mejora y diversificación del aprendizaje y la enseñanza de lenguas en los sistemas educativos de la Unión, donde se habría apuntado a la promoción de la diversidad lingüística como uno de los principales desafíos planteados en el terreno educativo ( $D O$ № $C$ 207, 12-8-1995). Las finalidades asignadas a la educación en lenguas en el Libro blanco se sintetizan "grosso modo" en tres: 1) incremento de las oportunidades económicas, 2) desarrollo del sentido de pertenencia e identidad, y 3) progreso educativo para el individuo.

Como atestiguan los anteriores documentos, el aprendizaje de idiomas constituye uno de los pilares básicos de la política educativa europea al que siguen aludiendo de manera casi inexcusable los textos comunitarios, presentándolo como un elemento favorecedor de la integración y la cohesión social. La adquisición de destrezas comunicativas en lenguas extranjeras ha de extenderse más allá de lo que son las etapas de escolarización, manteniéndose como un reto permanente para la población adulta. En lo que respecta a los sistemas educativos de los países miembros, las medidas recomendadas apuntan al comienzo del aprendizaje de una primera lengua extranjera a edades tempranas y a la introducción de una segunda lengua en etapas posteriores.

Más recientemente, las Conclusiones de la Presidencia del Consejo de Lisboa de marzo de $2000^{2}$ incluyen los idiomas como una de las nuevas competencias básicas que deben poseer todos los ciudadanos y ciudadanas europeos, junto con la cualificación en tecnologías de la información y de la comunicación, la cultura tecnológica, el espíritu empresarial y las habilidades para la socialización. A la vista de lo anterior, no resulta extraño que ese mismo año se publique una decisión por la que se declara el 2001 como "Año europeo de las lenguas" (Decisión 1934/2000/CE de 17 de julio de 2000, DO L 232 de 14-9-2000). En este texto se alude al aprendizaje de lenguas -al que se atribuyen ventajas humanas, culturales, políticas y también económicas- como instrumento que ayuda a bmar conciencia de la diversidad cultural y contribuye a la erradicación de la xenofobia, el racismo, el antisemitismo y la intolerancia. La mejora del conocimiento de idiomas sigue presente en el Informe de la Comisión Europea sobre los futuros objetivos precisos de los sistemas educativos y de formación (COM 200159 final), incluyéndolo en el objetivo más general de abrir esos sistemas al mundo. En el programa de trabajo presentado al Consejo Europeo de Barcelona (15 y 16 de marzo de 2002) se recogen dos cuestiones clave: animar a toda persona a aprender dos o incluso más lenguas, además de la materna; y animar a los centros escolares y de formación a emplear metodologías que motiven al alumnado a seguir estudiando idiomas en etapas posteriores de su vida.

\footnotetext{
${ }^{2}$ Conclusiones de la Presidencia. Consejo Europeo de Lisboa, 23 y 24 de marzo de 2000, disponible en http://europa.eu.int/european council/conclusions/index es.htm
} 
Concluiremos esta breve revisión de documentos con el plan de acción relativo a la promoción del aprendizaje de lenguas y de la diversidad lingüística establecido por la Comisión Europea para el bienio 2004-2006 (COM 2003449 final, 24-7-2003³), que aborda en uno de sus apartados la formación del profesorado. De conformidad con este texto, al profesorado de lenguas le corresponde desempeñar un papel crucial en la creación de una Europa multilingüe, estando llamado a ilustrar valores europeos como la apertura hacia los otros, la aceptación de las diferencias y la disposición a comunicar. Para ello, necesita disponer de una adecuada experiencia en la utilización de la lengua extranjera y comprender la cultura asociada a ella. Entre las medidas a adoptar para favorecer esta meta se apuntan las siguientes: 1) todo profesor o profesora de lenguas extranjeras debería haber efectuado una estancia prolongada en uno de los países donde se habla la lengua que enseña, así como poder actualizar sus conocimientos regularmente; 2) aunque los estados miembros sigan siendo los responsables de su formación inicial y continua, la acción de éstos podría completarse con los programas comunitarios como Sócrates o Leonardo; 3) la formación inicial debería proporcionarle un conjunto de capacidades y técnicas prácticas mediante la formación en el aula. Al mismo tiempo, debería poder actualizar sus capacidades lingüísticas y pedagógicas mediante el aprendizaje electrónico y a distancia; 4) se necesita favorecer los contactos entre profesionales y crear redes a nivel regional, nacional y europeo; 5) es necesario difundir entre estos profesionales los resultados de la investigación en el campo de la enseñanza de las lenguas extranjeras y las experiencias innovadoras, así como los ejemplos de buenas prácticas. Se necesita, además, prestar más atención al papel a desempeñar por los formadores del profesorado de lenguas e inspectores.

\section{3. $\quad$ LA DIMENSIÓN INTERCULTURAL EN LA ENSEÑANZA DE LAS LENGUAS}

En los últimos años los enfoques comunicativos han dominado -y siguen haciéndolo en buena medida- el panorama del aprendizaje de idiomas. Los defensores de una enseñanza más integrada de lengua y cultura suelen dirigir sus críticas hacia ellos, reprochándoles el haber privilegiado una concepción instrumental de la lengua -centrada en exclusiva en la adquisición de competencias lingüísticas- en detrimento de la dimensión cultural, que se habría visto reducida a un valor puramente anecdótico y no constituiría en sí misma un objetivo explícito del proceso de enseñanza-aprendizaje.

En base a estas críticas, se encuentra una nueva concepción del hecho comunicativo que pone el acento en el papel de los individuos como actores sociales dotados de múltiples identidades -nacional, local, regional, profesional, etc.- y de un bagaje de conocimientos sobre el mundo y de aptitudes y destrezas que entran en juego en la comunicación. Según se recoge en el Marco de referencia europeo para el aprendizaje, la enseñanza, y la evaluación de las lenguas, "La comunicación apela al ser humano en su totalidad ... Como agente social, cada individuo establece relaciones con un amplio conjunto de grupos sociales superpuestos, que unidos definen la identidad" (Instituto Cervantes, 2002, p. 12). La lengua no sólo es parte de la cultura, sino también el vehículo fundamental a través del cual se expresan las prácticas culturales y creencias de los grupos sociales. De ahí que todo intercambio comunicativo lleve aparejada una dimensión cultural. Aunque el principal interés de la enseñanza de una lengua siga siendo la comunicación en esa lengua, ésta no se circunscribe a la cuestión práctica de la competencia

\footnotetext{
${ }^{3}$ Disponible en http://europa.eu.int/eur-lex/es/com/cnc/2003/com2003 0449es01.pdf
} 
lingüística, sino que abarca también la relación entre la lengua y las prácticas culturales y creencias de un grupo, ya que estas últimas también desempeñan un papel en las interacciones comunicativas.

Tradicionalmente, en los enfoques comunicativos el modelo a seguir a la hora de enseñar una lengua era el del hablante nativo. Se entendía que el objetivo a alcanzar por el alumnado era la adquisición de un grado de competencia lingüística que se aproximase lo más posible al que pudiese tener una persona originaria del país. Desde la perspectiva de una enseñanza integrada de lengua y cultura se cuestiona este modelo, pues aunque un hablante no nativo pudiese quizá alcanzar, a base de mucho esfuerzo, unas destrezas lingüísticas que lo asemejasen al nativo, culturalmente nunca podría identificarse con él, ni tampoco es deseable que lo haga. En su lugar se apuesta por el hablante intercultural (Byram, 1997; Byram y Risager, 1999; Byram y Zarate, 1997; Kramsch, 2001).

Como se especifica en el Marco de referencia europeo (Instituto Cervantes, 2002), el modelo del hablante nativo deja de ser válido cuando la finalidad perseguida es desarrollar en el alumnado una competencia plurilingüe y pluricultural. Esta última se define como "la capacidad de utilizar las lenguas para fines comunicativos y de participar en una relación intercultural en que una persona, en cuanto agente social, domina -con distinto grado- varias lenguas y posee experiencias de varias culturas" ( $p$. 162). Esto no quiere decir que exista un conjunto de competencias diferenciadas que se superponen 0 se yuxtaponen, sino que existe una única competencia compleja de la que el hablante se sirve en sus intercambios comunicativos. Toda persona posee una competencia lingüístico-comunicativa única que se va ampliando progresivamente a lo largo de toda la vida, a medida que expande el círculo de sus contactos con la lengua. Del mismo modo, as distintas culturas a las que accede en el curso de su existencia (nacional, regional, social) van conformando una competencia pluricultural, uno de cuyos componentes -en interacción con todos los demás- es justamente la competencia plurilingüe. Un enfoque intercultural de la enseñanza de idiomas fija, como uno de sus objetivos fundamentales, promover el desarrollo armonioso de la personalidad del alumnado y de su sentimiento de identidad como respuesta a la enriquecedora experiencia que supone el encuentro con la alteridad en los ámbitos de la lengua y de la cultura.

Partiendo de las anteriores premisas, el Marco establece en su capítulo 5 una distinción entre dos grandes grupos de competencias que entrarían en juego en toda comunicación: competencias generales y competencias específicamente lingüísticas. Las competencias generales, donde se encuentran las que remiten de manera más directa a la dimensión intercultural, se subdividen en cuatro subcompetencias: conocimiento declarativo o saber, destrezas y habilidades o saber hacer, competencia existencial o saber ser y, por último, saber aprender. De estas subcompetencias, son las dos primeras las que guardan un vínculo más estrecho con la dimensión intercultural. Así, dentro del conocimiento declarativo o saber se incluyen el conocimiento del mundo o cultura general, el saber sociocultural y la toma de conciencia intercultural, mientras que las destrezas y habilidades interculturales corresponden al saber hacer. El conocimiento que el alumnado pueda tener cel país o países donde se habla la lengua objeto de aprendizaje en lo que respecta a datos geográficos, demográficos, económicos y políticos entra dentro de su conocimiento del mundo. El saber sociocultural -entendido como el conocimiento de la sociedad y de la cultura de la comunidad o comunidades que hablan una lengua dada- es también un aspecto del conocimiento del mundo, pero merece una atención especial, dado que frecuentemente no 
forma parte de las experiencias previas del alumnado y está deformado por estereotipos. La toma de conciencia intercultural, por su parte, se identifica con el conocimiento y la comprensión de las similitudes y diferencias existentes entre el propio universo cultural y el de la comunidad o comunidades objeto de estudio, incluyendo la toma de conciencia de la diversidad regional y social de ambos universos. Además del conocimiento objetivo, la conciencia intercultural engloba la toma de conciencia del modo en que cada comunidad es contemplada desde la óptica de los demás, frecuentemente caracterizado por los estereotipos. Por último, las destrezas y habilidades interculturales remiten a cuatro capacidades: la capacidad de establecer relaciones entre la cultura de origen y la extranjera; la sensibilidad cultural y la capacidad de emplear estrategias variadas para establecer contacto con personas de otras culturas; la capacidad de desempeñar el papel de intermediario cultural entre la propia cultura y la extranjera y de resolver situaciones de conflicto y malentendidos culturales; y la capacidad para superar estereotipos.

Descartado el modelo del hablante nativo por sus insuficiencias en el terreno cultural, la dimensión intercultural cifra su meta principal en convertir a las personas que aprenden una lengua en hablantes o mediadores interculturales capaces de introducirse en un marco complejo y en un contexto de identidades múltiples, así como de evitar los estereotipos que acompañan generalmente la percepción del otro al asignarle una única identidad: "El 'hablante intercultural' es una persona que tiene conocimientos de una, o preferentemente de más culturas e identidades sociales y que disfruta de la capacidad de descubrir y de relacionarse con gente nueva de otros entornos para los que no ha sido formado de forma intencional" (Byram y Fleming, 2001, p. 16). En el enfoque intercultural, el buen estudiante no es el que imita al nativo, sino el alumno que es consciente de sus propias identidades y culturas, de cómo los otros las perciben, y que conoce también las identidades y culturas de las personas con las que interactúa. De ahí que una enseñanza de las lenguas respetuosa con la dimensión intercultural deba contemplar, junto con el tradicional objetivo de adquirir la competencia lingüística necesaria para utilizar la lengua en cualquier comunicación oral o escrita según los códigos establecidos, un segundo objetivo más novedoso, desarrollar la competencia intercultural en la persona que aprende.

Byram y Risager (1999, p. 58) proponen una definición de la dimensión cultural en la enseñanza y aprendizaje de lenguas que incluye tres elementos interrelacionados. Dos de ellos conciernen al aprendizaje, mientras que el tercero afecta a la enseñanza. Así, la dimensión cultural se refiere a: 1) aquel aspecto de la competencia comunicativa que pone a la persona que aprende en contacto con el mundo cultural de un grupo particular de hablantes nativos; y 2) la capacidad de reflexionar, de analizar la propia cultura desde una perspectiva externa y de comprender su relación con otras culturas con el fin de facilitar la comunicación. En consonancia con estos dos elementos, el papel de la persona que aprende es el de un mediador entre culturas y es la mediación la que permite una comunicación efectiva. El tercer elemento a que aludíamos, relativo a la enseñanza, tiene que ver también con la mediación, pero podría llamarse la "profesionalización" de la mediación, ya que apela a la capacidad y responsabilidad del profesorado de lenguas de ayudar a quienes aprenden a comprender a los otros y la alteridad como base para la adquisición de una competencia comunicativa y cultural. El profesorado es, por consiguiente, un mediador profesional entre quienes aprenden y las lenguas y culturas extranjeras.

De acuerdo con Byram, Gribkova y Starkey (2002), los componentes de la competencia intercultural a desarrollar por un hablante/ mediador intercultural serían los siguientes: 
- Los puntos de vista y perspectivas interculturales ("saber ser"): apertura, capacidad para revisar la propia desconfianza frente a otras culturas y la fe en la de uno mismo. Se trata de una voluntad de relativizar los propios valores, creencias y comportamientos, aceptando que no son los únicos posibles, y de aprender a considerarlos desde la perspectiva de una persona exterior, de alguien que tiene un conjunto de valores, creencias y comportamientos distintos.

- El conocimiento ("saberes"): conocimiento de los distintos grupos sociales, de sus productos y de sus prácticas, tanto en el propio país como en el del interlocutor; conocimiento de las interacciones generales entre sociedades e individuos.

- Las capacidades de comparación, de interpretación y de establecer relaciones ("saber comprender"): capacidad general para interpretar un documento o un acontecimiento vinculado a otra cultura, para explicarlo y ponerlo en relación con documentos o acontecimientos vinculados a la propia cultura.

- Las capacidades de descubrimiento y de interacción ("saber aprender/ hacer"): capacidad para adquirir nuevos conocimientos sobre una cultura y unas prácticas culturales dadas, así como para manejar conocimientos, puntos de vista y capacidades sometiéndose a las normas de la comunicación y de la interacción en tiempo real.

- La visión crítica en el plano cultural ("saber comprometerse”): capacidad para evaluar, de manera crítica, los puntos de vista, prácticas y productos del propio país y de las otras naciones y culturas.

Es evidente que el proceso de adquisición de una competencia intercultural no puede darse nunca por concluido y que ésta no tiene que ser perfecta para permitir una comunicación satisfactoria, ya que las identidades y valores sociales de los individuos no son algo fijo e inmutable, sino que se van modificando de manera continua a lo largo de la vida, a medida que se entra en contacto con nuevos grupos.

De los componentes de la competencia intercultural merece la pena detenerse en el último, la visión crítica en el plano cultural. Byram (1997, pp. 63-64) insiste en la importancia de desarrollar en el alumnado una conciencia cultural crítica. Según hemos visto anteriormente, se trataría de capacitar a este último para evaluar de manera crítica y sobre la base de criterios precisos las perspectivas, las prácticas y los productos de la propia cultura y de la de otros países. Este ejercicio requiere desarrollar una capacidad para: 1) identificar e interpretar los valores explícitos o implícitos de los documentos y acontecimientos de la propia cultura y de otras, utilizando aproximaciones analíticas que permitan situar un documento o acontecimiento en su contexto y tomar conciencia de su dimensión ideológica; 2) proceder a una evaluación de los documentos y acontecimientos adoptando una perspectiva clara, basándose para ello en criterios precisos como los derechos humanos, la democracia liberal, la religión o la ideología política; y 3) actuar en calidad de mediador en los intercambios culturales, siendo consciente del conflicto que puede crearse entre las posiciones ideológicas propias y las de los otros y tratando de encontrar criterios comunes. Para Starkey (2003), el desarrollo de esta conciencia debería iniciarse desde el momento en que se comienza a aprender una lengua y continuar durante todo el proceso de aprendizaje, lo que conllevaría importantes consecuencias para las escuelas. En su opinión, el marco de 
referencia para establecer comparaciones entre los comportamientos, prácticas e instituciones de la cultura del alumnado y la extranjera podría venir dado por el conocimiento y comprensión de los derechos humanos.

\section{PAPEL DEL PROFESORADO EN EL DESARROLLO DE LA DIMENSIÓN INTERCULTURAL}

A la vista de los planteamientos anteriores, cabe preguntarse cuál debe ser el papel del profesorado a la hora de desarrollar esa dimensión intercultural. Es importante precisar que el objetivo de la enseñanza de la mencionada dimensión no es tanto la simple transmisión de informaciones sobre un país dado como la búsqueda del desarrollo de capacidades, perspectivas y puntos de vista. Podríamos decir que remite más bien al mundo de los valores y de las actitudes. Desde esta óptica, la misión esencial del docente es hacer captar al alumnado la relación entre la cultura propia y las otras culturas, de suscitar en ese alumnado una curiosidad por la alteridad y de hacerle tomar conciencia del modo en que otros pueblos e individuos lo perciben a él y a su cultura. Se convierte así, como hemos apuntado anteriormente, en mediador entre dos o más culturas. Desempeñar la labor de mediación profesional forma parte de la tarea de enseñar lenguas, siendo una responsabilidad a la que ha de prestarle especial atención en los niveles de escolarización obligatoria. Además, como apuntan Byram y Risager (1999, p. 76), la potenciación de la dimensión cultural implica el establecimiento de nuevas relaciones entre profesorado y alumnado: al primero se le atribuye el papel de consejero, de guía, y no de simple transmisor de conocimientos, mientras que al segundo le corresponde el trabajo autónomo sobre el propio material.

Entre las tareas que competen al profesorado en relación con el alumnado figuran las siguientes: a) prepararlo para entablar relaciones con personas de otras culturas; b) favorecer que comprenda y acepte a esas personas diferentes como individuos que tienen distintos puntos de vista, valores y comportamientos; c) ayudarle a comprender el funcionamiento de las interacciones culturales; d) mostrarle que las identidades sociales son parte integrante de cualquier relación; e) demostrarle la influencia de la percepción que se tiene de los otros y de la visión que los otros tienen de uno mismo en el éxito de la comunicación; f) conducirle a saber más, por sí mismo, de las personas con las que se comunica; y g) ayudarle a aprehender el carácter enriquecedor de este tipo de experiencias y relaciones (Byram, Gribkova y Starkey, 2002).

Articular el trabajo del aula alrededor de la dimensión intercultural implica dar prioridad a procedimientos como la comparación, la identificación con la alteridad (ponerse en el lugar del otro) y la observación de la propia cultura. Incluimos aquí, a título de sugerencia, algunas indicaciones prácticas que favorecerían la orientación del trabajo a desarrollar en las clases en esa dirección:

- Trabajar con materiales auténticos extraídos de la comunidad o comunidades objeto de estudio.

- Establecer vínculos con escuelas e institutos extranjeros a través de Internet y/o utilizando el correo electrónico. 
- Entrar en contacto con hablantes nativos en el propio país y, si es posible, en el extranjero, empleando también las vías arriba indicadas.

- Identificarse con las perspectivas y experiencias de las personas que habitan los países y comunidades donde se habla la lengua objeto de estudio, recurriendo a simulaciones, juegos de rol, etc.

- Investigar e informarse sobre algún aspecto particular de las comunidades o países donde se habla la lengua extranjera.

- Planificar un viaje al extranjero, aunque sólo sea como ejercicio.

- Comparar, de manera general y específica, el propio país y las comunidades o países donde se habla la lengua estudiada.

A la hora de poner en práctica las diferentes tareas sugeridas, el profesorado debe fomentar el trabajo en grupo, el diálogo, el debate y la participación activa y reflexiva. Especialmente importante, además, es la utilización de una gama lo más amplia posible de fuentes de información: textos originales, grabaciones de audio, cartas, fotografías, gráficos, dibujos, etc. En esta tarea de búsqueda de materiales complementarios debe procurarse implicar al propio alumnado. Por otra parte, de cualquier texto o documento que se vaya a utilizar, es importante proporcionar dos tipos de información: el contexto (fecha, tipo y lugar de publicación, público al que se dirige, acontecimientos externos que han podido influir en su concepción, tendencia religiosa, política, económica o cultural implícita) y la intención (¿se pretende persuadir, argumentar, hacer publicidad de algo, etc.).

\section{IMPLICACIONES PARA LA FORMACIÓN DEL PROFESORADO}

El nuevo contexto derivado de la integración europea, del incremento de los intercambios internacionales y de la presencia cada vez mayor en nuestras sociedades de personas pertenecientes a distintas culturas y hablantes de diferentes lenguas demanda un nuevo perfil docente que dé respuesta a las necesidades planteadas en lo que atañe a los objetivos formativos atribuidos al aprendizaje de idiomas. En una Europa plurilingüe y multicultural, el profesorado debe convertirse en un agente de cambio, continuamente innovador y consciente de la evolución de su alumnado y de su entorno. Entre los nuevos roles que se le asignan figura el de educar ciudadanos y ciudadanas europeos en un espíritu de tolerancia, de comprensión y de respeto hacia la diversidad, algo que requiere una adecuada formación para poder ejercer ese papel de mediador entre lenguas y culturas.

Los anteriores planteamientos conllevan abandonar lo que nosotros calificaríamos como una visión más bien técnica de la misión del profesorado de lenguas, según la cual su objetivo era que el alumnado adquiriese un conjunto de destrezas o habilidades puramente lingüísticas, para adoptar un enfoque más educativo o formativo, en el que esa adquisición de destrezas, ampliada a capacidades que van más allá del aprendizaje de la gramática, la fonética y el vocabulario, deviene un instrumento al servicio de una meta educativa más importante: desarrollar el espíritu crítico y promover la comprensión de las diferencias culturales como elemento-clave para mejorar el entendimiento entre los seres humanos. 
Pensamos que, en los últimos años, la cultura ha estado presente en las aulas a través del trabajo sobre documentos auténticos o, en la mayoría de los casos, falsamente auténticos -a veces incluidos en los propios manuales- cuya finalidad era introducir en la clase situaciones comunicativas lo más cercanas posibles a las que podrían producirse en situaciones reales en el país extranjero. A través de estos documentos se acercaba al alumnado al contexto social y cultural de ese país, pero el objetivo nunca era promover la reflexión sobre esa realidad sociocultural y mucho menos la adquisición de una conciencia crítica que favoreciese la relativización de las diferencias culturales. El trabajo sobre la cultura -entendido de una manera limitada como adquisición de conocimientos sobre el país extranjero necesarios o útiles para fines comunicativos- quedaba en buena medida a merced de la buena disposición o voluntad del profesorado. Asumir una perspectiva intercultural de la enseñanza de las lenguas implica reorientar por completo el trabajo del aula.

El cambio de foco de atención en la naturaleza y objetivos del aprendizaje de lenguas tiene así importantes implicaciones para la práctica cotidiana del profesorado, que se verá enfrentado a nuevas tareas que requerirán la incorporación del enfoque intercultural en su formación inicial y continua. Más concretamente, entre las demandas que se le plantean figuran las siguientes: 1) incrementar su propia conciencia cultural y su competencia intercultural; 2) cambiar o adaptar sus métodos para promover las anteriores capacidades en el alumnado; y 3) tener presente su cambio de identidad profesional, de docente de lenguas a docente de comunicación intercultural.

Edelhoff (cit. en Sercu, 2001, pp. 255-256) ofrece una relación detallada de las cualidades que debe desarrollar el profesorado de lenguas para llevar a cabo una enseñanza intercultural; cualidades que, a nuestro juicio, deberían tenerse en cuenta en la planificación de su formación inicial y continua. Las cualidades - de las que presentamos una síntesis- se desglosan en actitudes, conocimientos y destrezas. Las incluidas en el plano de las actitudes se identifican en buena medida con las que debe promover en el alumnado: 1) considerar cómo lo ven otras personas y tener curiosidad por conocer más sobre sí mismo y sobre los otros; 2) estar dispuesto a experimentar y negociar para alcanzar un entendimiento; 3) estar dispuesto a compartir significados, experiencias y afectos con personas de otros países y con el propio alumnado; 4) estar dispuesto a participar activamente en la búsqueda de las aportaciones de los idiomas al entendimiento internacional; y 5) adoptar la función y el papel de intérprete social e intercultural, y no de embajador de la cultura extranjera. En el terreno de los conocimientos, se le exige: 1) tener conocimientos y estudiar más sobre la historia cultural de los países y comunidades que utilizan la lengua estudiada; 2) tener conocimientos y estudiar más sobre su propio país y sobre el modo en que los otros lo perciben; 3) disponer de un conocimiento activo que pueda convertir en accesible la situación de aprendizaje; y 4) conocer cómo funciona la lengua en la comunicación y cómo puede utilizarse de manera eficaz para la comprensión. Finalmente, el profesorado debe dominar y perfeccionar las siguientes destrezas: 1) destrezas de comunicación adecuadas para la negociación; 2) destrezas para procesar información en todo tipo de medios (audiovisuales, orales, escritos) y en las interacciones cara a cara; y 3) crear entornos de aprendizaje basados en la negociación y en la experiencia.

En nuestra opinión, la principal prioridad de la formación del profesorado desde una perspectiva intercultural no debe ser la adquisición de un saber complementario sobre uno o varios países extranjeros, sino la organización de las clases y la metodología a emplear en ellas. Para poder transmitir 
la dimensión intercultural, el profesorado necesita tener, más que muchos conocimientos de otros países y otras culturas, la capacidad de crear en el aula las condiciones necesarias para lograr el compromiso personal del alumnado en los planos intelectual y emocional, capacidad que se adquiere a través de la práctica y la reflexión. El propio profesorado debe convertirse en "aprendiz intercultural". Se plantea así, además, la necesidad de ampliar su centro de atención tradicional en las lenguas que enseña y en los países diferentes al propio para incluir un interés por la diversidad en el propio país y por la naturaleza multilingüe y multicultural de los países donde se habla la lengua objeto de aprendizaje.

Teniendo en cuenta la importancia que reviste la utilización de unos materiales adecuados y variados a la hora de desarrollar la dimensión intercultural en las clases, consideramos especialmente necesario incluir en la formación del profesorado el análisis crítico de materiales curriculares, especialmente de los libros de texto. Se requiere examinar hasta qué punto la imagen de la cultura extranjera que ofrecen estos últimos es representativa, completa, realista, está actualizada y da cuenta de su pluralidad interna. En el caso de que un manual presente una visión única y directiva de las cosas, una visión de la cultura extranjera cerrada y definitiva, el profesorado debe hacer ver a su alumnado que existen otras perspectivas e impulsarlo a analizar el libro (dibujos, fotografías y elementos escritos) de manera crítica.

En otro orden de cosas, dado que el enfoque a adoptar a la hora de desarrollar la dimensión intercultural no se centra tanto en la transmisión de informaciones culturales como de capacidades analíticas, Byram, Gribkova y Starkey (2002) señalan la conveniencia de contemplar, dentro de la formación general del profesorado de idiomas, una formación específica en el análisis de los sistemas culturales.

Concluiremos apuntando, a modo de síntesis, los tres pilares básicos en que debe fundamentarse la formación del profesorado de acuerdo con Byram (2003): 1) promover el plurilingüismo (entendido éste en el sentido del Marco de referencia europeo), 2) centrar la atención en la competencia intercultural y 3) preparar al profesorado para comprometerse con la educación en valores y la ciudadanía democrática.

\section{BIBLIOGRAFÍA}

BUTTJES, D. y BYRAM, M. (Eds.) (1991): Mediating Languages and Cultures. Clevedon. Multilingual Matters.

BYRAM, M. (1997): Teaching and Assessing Intercultural Communicative Competence. Clevedon. Multilingual Matters.

BYRAM, Michael (2003): "Teacher education -visions from/in Europe". Babylonia, 3-4, pp. 7-10. Consultado el 10-4-2004 en www.babylonia-ti.ch/BABY3 403/PDF/byr.pdf

BYRAM, M.; GRIBKovA, B. y StARKEY, H. (2002): Développer la dimension interculturelle de l'enseignement des langues. Une introduction à l'usage des enseignants. Strasbourg. Conseil de l'Europe.

BYRAM, M. y RISAGER, K. (1999): Language Teachers, Politics and Cultures. Clevedon. Multilingual Matters. 
Byram, M. y Fleming, M. (2001): Perspectivas interculturales en el aprendizaje de idiomas. Enfoques a través del teatro y la etnografía. Madrid. Cambridge University Press.

BYRAM, M. y ZARATE, G. (1997): "Définitions, objectifs et évaluation de la compétence socioculturelle". En M. BYRAM, G. ZARATE y G. NEUNER: La compétence socioculturelle dans l'apprentissage et l'enseignement des langues. Strasbourg. Conseil de l'Europe, pp. 7-36.

COMISIÓN EUROPEA (1993): Libro verde sobre la dimensión europea de la educación. Luxemburgo. Oficina de publicaciones de las Comunidades Europeas.

COMISIÓN EUROPEA (1995): Libro blanco sobre la educación y la formación. Enseñar y aprender. Hacia la sociedad del conocimiento. Luxemburgo. Oficina de publicaciones de las Comunidades Europeas.

COMISIÓN EUROPEA (1999): Unión Europea. Recopilación de los Tratados. Tomo I, Vol l Luxemburgo. Oficina de publicaciones oficiales de las Comunidades Europeas.

EURYDICE (2001): La enseñanza de las Lenguas Extranjeras en el contexto escolar europeo. Madrid. MECD. Secretaría General de Educación y Formación Profesional. CIDE.

INSTITUTO CERVANTES (2002): Marco de referencia europeo para el aprendizaje, la enseñanza y la evaluación de lenguas. Madrid. Instituto Cervantes. Versión electrónica en http://cvc.cervantes.es/obref/marco, traducción y adaptación española del Common European Framework of Reference for Languages: Learning, Teaching, Assessment. Council of Europe, 2001.

KRAMSCH, C. (1993): Context and Culture in Language Teaching. Oxford. Oxford University Press.

KRAMSCH, C. (2001): "El privilegio del hablante intercultural". En M. BYRAM y M. FLEMING: Perspectivas interculturales en el aprendizaje de idiomas. Enfoques a través del teatro y la etnografía. Madrid. Cambridge University Press, pp. 23-37.

SERCU, L. (2001): "Formación de profesores en ejercicio y adquisición de competencia intercultural". En M. ByRAm y M. Fleming: Perspectivas interculturales en el aprendizaje de idiomas. Enfoques a través del teatro y la etnografía. Madrid. Cambridge University Press, pp. 254-286.

STARKEY, H. (2003): "Compétence interculturelle et éducation àla citoyenneté démocratique: incidences sur la méthodologie d'enseignement des langues". En M. BYRAM (Coord.): La compétence interculturelle. Srasbourg. Conseil de l'Europe, pp. 67-88.

ZÁRATE (1986): Enseigner une culture étrangère. Paris. Hachette. 


\title{
Contactar
}

Revista lberoamericana de Educación

\author{
Principal OEI
}

